\title{
JOINING NON-LOW C.E. SETS WITH DIAGONALLY NON-COMPUTABLE FUNCTIONS
}

\author{
LAURENT BIENVENU, NOAM GREENBERG, ANTONÍN KUČERA, JOSEPH S. MILLER, \\ ANDRÉ NIES, AND DAN TURETSKY
}

\begin{abstract}
We show that every non-low c.e. set joins all $\Delta_{2}^{0}$ diagonally noncomputable functions to $\emptyset^{\prime}$. We give two proofs: a direct argument, and a proof using an analysis of functions that are DNC relative to an oracle, extending work by Day and Reimann. The latter proof is also presented in the language of Kolmogorov complexity.
\end{abstract}

\section{INTRODUCTION}

Computability theorists often identify classes of oracles that are considered either weak or strong. In this paradigm, the computable sets are the ultimately weak sets, as they contain no information, and the halting problem $\emptyset^{\prime}$ is a paragon of strength, certainly when comparing it to other computable enumerable (c.e.) sets. Thus, an oracle that shares some of the properties of the computable sets is considered weak (and we think of the oracle as "close to being computable"), while on the other hand, an oracle that in some way resembles the halting problem is considered strong.

A canonical example is given by the jump operator. Soare [16] defined a set $A$ to be low if $A^{\prime} \equiv_{\mathrm{T}} \emptyset^{\prime}$ : the Turing degree of the halting problem relative to $A$ is as low as possible. A low set is indistinguishable from computable sets as far as the jump operator is concerned, and so is considered weak. Dually, a set $A$ is high if $A^{\prime} \geqslant_{\mathrm{T}} \emptyset^{\prime \prime}$, equivalently, by a result of Martin's, if it computes a function that dominates all computable functions. This is an example of a notion of strength: a high set is a set that can solve a problem (dominate computable functions) that the halting problem can solve, but computable sets, and sets close to being computable, cannot solve.

In this paper we consider another notion of strength: computing a diagonally non-computable (DNC) function. A function $g: \omega \rightarrow \omega$ is DNC if for a fixed universal partial computable function $J$, for all $e \in \operatorname{dom} J$, we have $g(e) \neq J(e)$. A Turing degree is a $D N C$ degree if it contains a DNC function; it is not difficult to see that the DNC degrees are closed upwards, as we can find infinite computable sets disjoint from the domain of $J$, and use them for coding.

Last compilation: September 29, 2014.

2010 Mathematics Subject Classification. Primary 03D25; Secondary 03D28, 03D32.

Miller is supported by the National Science Foundation under grant DMS-1001847. Greenberg was supported by the Royal Society of New Zealand via the Marsden Fund and a Rutherford Discovery fellowship. Nies was supported by the Marsden Fund. Turetsky was supported by the Marsden Fund, via a postdoctoral scholarship and by FWF grant P23989-N13. Some of the work by Bienvenu, Greenberg, Kučera, Nies and Turetsky was performed during a research-in-pairs stay at the Mathematisches Forschungsinstitut Oberwolfach. 
Certainly the Turing degree $\mathbf{0}^{\prime}$ of the halting problem is a DNC degree, since $\emptyset \emptyset^{\prime}$ can tell whether $e \in \operatorname{dom} J$ or not. The study of DNC degrees (started by Jockusch in [6]) was originally motivated by their coincidence with those degrees that can compute a fixed-point-free function, a function $f$ such that $W_{f(e)} \neq W_{e}$ for all $e$. Thus, the recursion theorem shows that computable sets do not have DNC degree. Another important aspect of DNC degrees is that they are a superclass of a more restrictive notion of oracular strength, that of having $P A$ degree. For the connection, we mention that a Turing degree is a PA degree if and only if it contains a $D N C$ set, i.e., a $\{0,1\}$-valued $\mathrm{DNC}$ function.

Often, notions of strength and weakness that measure different aspects of computational power do not cohere. For example, there is a low PA degree; this degree is weak in one sense and strong in another. Thus, it is interesting when we observe meaningful interactions between notions of strength, in particular when these interactions involve Turing reducibility. In this way, we make a connection between relative and absolute notions of strength and weakness. This is certainly the case when considering how PA and DNC degrees interact with the c.e. sets. As a first example, in contrast with the low PA degree mentioned above, Arslanov [1] showed that among the c.e. degrees, only the top one $\mathbf{0}^{\prime}$ is a DNC degree. This was extended to the $n$-REA hierarchy in [7], see also [17].

On the other hand, $\Delta_{2}^{0}$ DNC degrees resemble $\mathbf{0}^{\prime}$ also in that each of them lies above a nonzero c.e. degree ([10],[11]). The methods that are behind this phenomenon provide an alternative approach (priority-free) to a solution to Post's problem and some other finite injury priority constructions. The basic idea is to guarantee some properties of a c.e. set by properties of some set of PA degree in which the c.e. set is coded. Incompleteness and avoiding an upper cone are illustrative examples. However, in some aspects, $\Delta_{2}^{0} \mathrm{PA}$ degrees are limited compared to all PA degrees. For example, there is no minimal pair of $\Delta_{2}^{0} \mathrm{PA}$ (or even DNC) degrees [11]. Oracles slightly stronger than $\emptyset^{\prime}$ are required to construct minimal pairs of PA degrees; in [11] it was shown that $\mathbf{0}^{\prime}$ is the greatest lower bound of the set of joins of minimal pairs of PA degrees.

All of this led to a question whether given an incomplete c.e. set $A$ there is a PA set $X$, or more generally, a DNC function $f$, such that $A<_{\mathrm{T}} X<_{\mathrm{T}} \emptyset^{\prime}$, or $A<_{\mathrm{T}} f<_{\mathrm{T}} \emptyset^{\prime}$. In other words, can the incompleteness of a c.e set $A$ be witnessed by an incomplete $\Delta_{2}^{0}$ PA set or DNC function? And if not, which incomplete c.e. sets have this property?

A negative answer for the first question was given by Kučera and Slaman (unpublished), who constructed an incomplete c.e. set that joins all $\Delta_{2}^{0} \mathrm{DNC}$ functions to $\emptyset^{\prime}$. Their proof used the $\emptyset^{(3)}$-priority method. The focus thus turns to the characterization of c.e. degrees with that property. Recently, Day and Reimann [3] solved the problem for the class of PA degrees.

Theorem 1.1 (Day and Reimann). A c.e. set $A$ is low if and only if $A$ is computable from an incomplete, $\Delta_{2}^{0} \mathrm{PA}$ degree.

By the low basis theorem, every low c.e. set is computable from a low PA degree, and so the dichotomy is sharp. We extend their result to the wider class of diagonally non-computable functions.

Theorem 1.2. If $A$ is a non-low c.e. set and $f$ is a $\Delta_{2}^{0}$ diagonally non-computable function, then $\emptyset^{\prime} \leqslant_{\mathrm{T}} A \oplus f$. 
This result can be thought of another version of Arslanov's completeness criterion. The criterion says that being c.e. and being DNC are mutually exclusive properties; the theorem states that when non-lowness is added, these properties are complementary. Another interpretation of this theorem is that relativizing to a $\Delta_{2}^{0}$ DNC function $f$ eliminates the difference between all non-low c.e. degrees; to $f$, they all look like $\mathbf{0}^{\prime}$.

Theorem 1.2 gives the solution of the problem mentioned above for DNC degrees.

Corollary 1.3. A c.e. set $A$ is low if and only if $A$ is computable from an incomplete, $\Delta_{2}^{0}$ diagonally non-computable function.

The corollary is also of interest because very few characterizations of lowness are known, even among the c.e. degrees.

A weak version of Theorem 1.2 was first proved by Bienvenu, Greenberg, Kučera, Nies and Turetsky during a stay at the mathematics research institute in Oberwolfach (see Theorem 4.5). They gave two arguments: a direct one, and one using Kolmogorov complexity. Miller then proved Theorem 1.2. Kučera then found how to adapt the direct argument to prove the theorem. Below, we give both proofs, and also give a third proof, similar to the first, but which uses Kolmogorov complexity.

We note that all proofs are uniform: effectively, given a c.e. index for a non-low c.e. set $A$, and a $\Delta_{2}^{0}$ index for a DNC function $f$, we can compute an index of a Turing functional $\Psi$ such that $\emptyset^{\prime}=\Psi(A, f)$.

1.1. Definitions and notation. Some of the following definitions were also given in the introduction.

We fix a universal partial computable function $J$. The most common definition of $J$ is $J(e)=\varphi_{e}(e)$, where $\left\langle\varphi_{e}\right\rangle$ is an acceptable enumeration of all partial computable functions. What is important is the acceptability of $J$ : for every partial computable function $\psi$ there is an injective, total computable function $\alpha$ such that $\psi=J \circ \alpha$. An index for $\alpha$ is obtained effectively from an index for $\psi$.

A function $f \in \omega^{\omega}$ is diagonally non-computable if for all $e \in \operatorname{dom} J$ we have $f(e) \neq J(e)$. The class of diagonally non-computable functions is denoted DNC. We say that a set $D \in 2^{\omega}$ has DNC-degree if $D$ computes a diagonally non-computable function.

All of these notions can be relativized to an oracle. For an oracle $A$, we fix a universal $A$-partial computable function $J^{A}$. A function $f$ is DNC relative to $A$ if $f(e) \neq J^{A}(e)$ for all $e \in \operatorname{dom} J^{A}$. We identify $J$ with $J^{\emptyset}$.

For any set $A$, we let $A^{\prime}=\operatorname{dom} J^{A}$. This is a universal $A$-c.e. set. A c.e. set $A$ is low if $A^{\prime} \equiv_{\mathrm{T}} \emptyset^{\prime}$.

We let $\mathrm{DNC}_{2}=\mathrm{DNC} \cap 2^{\omega}$. This is the collection of $\mathrm{DNC}$ functions with values in $\{0,1\}$. Recall that a $\Pi_{1}^{0}$ class is an effectively closed subset of $2^{\omega}$ - the collection of infinite paths of a computable tree $T \subseteq 2^{<\omega}$. The class $\mathrm{DNC}_{2}$ is a $\Pi_{1}^{0}$ class. A set $A \in 2^{\omega}$ has PA degree if every nonempty $\Pi_{1}^{0}$ class contains an $A$-computable element. There are several equivalent definitions of PA degrees: a set A has PA degree if and only if it computes a consistent completion of Peano arithmetic, if and only if it computes some $\mathrm{DNC}_{2}$ set.

If $f$ and $g$ are natural-valued functions, then we write $f \leqslant^{+} g$ if for some constant $c$ we have $f(x) \leqslant g(x)+c$ for all $x$ in the common domain of $f$ and $g$. We write $f={ }^{+} g$ if $f \leqslant^{+} g$ and $g \leqslant^{+} f$. 
From algorithmic randomness we use the notion of prefix-free Kolmogorov complexity $K$ (we sometimes drop the term "prefix-free" for concision). We denote a universal prefix-free machine by $\mathbb{U}$, and a universal oracle prefix-free machine by $\mathbb{U}^{Z}$. A set $Z \in 2^{\omega}$ is 1 -random if and only if $K\left(Z \uparrow_{n}\right) \geqslant^{+} n$, where $Z \uparrow_{n}$ denotes the prefix of $Z$ of length $n$.

We use Lachlan's square bracket convention for evaluating compound expressions at a given stage. For example, if $\left\langle A_{s}\right\rangle$ is an effective enumeration of a c.e. set $A$ and $K_{s}^{Z}$ denotes the stage $s$-approximation of oracle prefix-free complexity $K^{Z}$, then we write $K^{A}[s]$ for $K_{s}^{A_{s}}$.

\section{Relatively DNC Degrees}

Using work by Day and Miller [2] that connects Levin's neutral measures to PA degrees, Day and Reimann [3] proved the following surprising theorem.

Theorem 2.1 (Day and Reimann). Suppose that $X \in 2^{\omega}$ has PA degree and that $C$ is a c.e. set. Then either $X \oplus C \geqslant_{\mathrm{T}} \emptyset^{\prime}$, or $X \geqslant_{\mathrm{T}} C$.

Later, Kučera and independently Miller found direct proofs of this theorem. We give one such proof.

Proof. Fix effective enumerations $\left\langle\emptyset_{s}^{\prime}\right\rangle$ and $\left\langle C_{s}\right\rangle$ of $\emptyset^{\prime}$ and of $C$. We let $A$ be the set of pairs $(e, i)$ of numbers such that $e$ enters $\emptyset^{\prime}$, and if $i$ enters $C$, it does not do so at an earlier stage. That is, $(e, i) \in A$ if there is a stage $s$ such that $e \in \emptyset_{s}^{\prime}$ but $i \notin C_{s-1}$. Let $B$ be the set of pairs $(e, i)$ such that for some $s, i \in C_{s}$ but $e \notin \emptyset_{s}^{\prime}$. The sets $A$ and $B$ are disjoint c.e. sets. Sets of PA degree compute separators for disjoint c.e. sets: there is a set $E \leqslant_{\mathrm{T}} X$ that contains $B$ and is disjoint from $A$. There are two cases.

Case 1. Assume that there is an $e \notin \emptyset^{\prime}$ such that for all $i \notin C$ we have $(e, i) \notin E$. Then $i \in C$ if and only if $(e, i) \in E$, so $E \geqslant_{\mathrm{T}} C$, indeed $E \geqslant_{m} C$.

Case 2. Otherwise, for all $e \notin \emptyset^{\prime}$, there is an $i \notin C$ such that $(e, i) \in E$. Note that if $e \in \emptyset^{\prime}$ and $i \notin C$, then $(e, i) \in A$ and so $(e, i) \notin E$. So we can enumerate $\omega \backslash \emptyset^{\prime}$ with oracle $E \oplus C$ by searching for $e \in \omega$ and $i \notin C$ such that $(e, i) \in E$. Therefore, $E \oplus C \geqslant_{T} \emptyset^{\prime}$.

As we noted in the proof, in Case 1 we actually get $E \geqslant_{m} C$. If $X \in \mathrm{DNC}_{2}$ then for any pair $A, B$ of disjoint c.e. sets there is a separator $E(E \supseteq B$ and $E \cap A=\emptyset)$ that is many-one reducible to $X$. For we can find an injective computable function $\alpha$ such that $J(\alpha(n))=1$ if $n \in A$ and $J(\alpha(n))=0$ if $n \in B$; we let $E=\alpha^{-1} X$. Thus, we get:

Porism 2.2. If $X \in \mathrm{DNC}_{2}$ and $C$ is c.e., then either $X \oplus C \geqslant_{\mathrm{T}} \emptyset^{\prime}$ or $X \geqslant_{m} C$. In particular, if $X$ is Turing incomplete and $X \geqslant_{\mathrm{T}} C$ then $X \geqslant_{m} C$.

Day and Reimann gave the following corollary of their theorem.

Theorem 2.3 (Day and Reimann). Assume that $X$ has PA degree and $C$ is a c.e. set. Either $X \oplus C \geqslant_{\mathrm{T}} \emptyset^{\prime}$ or $X$ has $\mathrm{PA}$ degree relative to $C$.

Proof. Apply Theorem 2.1 to $X$ and $C$. If $X \oplus C \geqslant_{\mathrm{T}} \emptyset^{\prime}$, we are done. Otherwise, $X \geqslant_{\mathrm{T}} C$ and $X \nexists_{\mathrm{T}} \emptyset^{\prime}$. The $\Pi_{1}^{0}$ class of pairs of sets $(A, B)$ such that $A \in \mathrm{DNC}_{2}$ and $B \in \mathrm{DNC}_{2}(A)$ is nonempty, and so we can find a $Y \leqslant_{\mathrm{T}} X$ of $\mathrm{PA}$ degree such that $X$ has PA degree relative to $Y$ (this argument was noted by Simpson [15, 
Theorem 6.5]). Since $X \geqslant_{\mathrm{T}} Y, Y \oplus C \leqslant_{\mathrm{T}} X$ and so $Y \oplus C \ngtr_{\mathrm{T}} \emptyset^{\prime}$. Applying Theorem 2.1 to $Y$ and $C$ gives $Y \geqslant_{T} C$. Therefore, $X$ has PA degree relative to $C$.

We cannot replace the condition that $X$ has PA degree in Theorem 2.1 with the weaker assumption that $X$ has DNC degree. To see this, we recall that every 1-random set has DNC degree.

Proposition 2.4 (Kučera [9]). Every 1-random set computes a DNC function.

Proof. It is convenient to fix an effective bijection between strings and natural numbers: identify $\sigma \in 2^{<\omega}$ with $n \in \omega$ if $1 \sigma$ is the binary expansion of $n+1$. Let $X$ be 1-random and define $g(n)=X\lceil n$.

If $g(n)=J(n)$, then $K\left(X\lceil n)={ }^{+} K(g(n))=K(J(n)) \leqslant{ }^{+} K(n) \leqslant+2 \log (n)\right.$, where the constant does not depend on $n$. So the fact that $X$ is 1-random implies that $g(n) \neq J(n)$ for all but finitely many $n$. By changing at most finitely many values of $g$, we get an $X$-computable DNC function.

Now let $C$ be a low c.e. set that is not $K$-trivial (for $K$-triviality see [4] or [12]). For example, one can take a low c.e. set that is not superlow; all $K$-trivial sets are superlow (Nies [13]). By the low basis theorem relative to $C$, there is a 1-random set $Z$ such that $Z \oplus C$ is low. So $Z \oplus C \Varangle_{\mathrm{T}} \emptyset^{\prime}$, and by Proposition 2.4, $Z$ has DNC degree. However, $Z \ngtr_{\mathrm{T}} C$, because a c.e. set computable from an incomplete random set must be $K$-trivial (Hirschfeldt, Nies and Stephan [5]). Therefore, we see that in Theorem 2.1 we cannot replace PA with DNC. Overall, the low sets that are not $K$-trivial form an interesting "grey zone": they are joined to $\mathbf{0}^{\prime}$ by some but not all $\Delta_{2}^{0}$ random degrees. On the other hand, every c.e. degree is low-cuppable in the $\Delta_{2}^{0}$ degrees, and so by relativizing the low basis theorem, is also PA-cuppable.

Note, however, that the application above of the relativized low basis theorem yielded a set that is random relative to $C$, and so has DNC degree relative to $C$. This is necessary, because in Theorem 2.3 we can replace PA by DNC.

Theorem 2.5. Assume that $X \in 2^{\omega}$ has $\mathrm{DNC}$ degree and that $C$ is a c.e. set. Then either $X \oplus C \geqslant_{\mathrm{T}} \emptyset^{\prime}$ or $X$ has $\mathrm{DNC}$ degree relative to $C$.

The same theorem with 1-random instead of PA or DNC was proved by Hirschfeldt, Nies and Stephan [5].

Proof. Let $g: \omega \rightarrow \omega$ be an $X$-computable DNC function. By the recursion theorem, we may assume that we control the values of the diagonal function $J$ at a computable sequence of positions $\left\{k_{n, m}\right\}_{n, m \in \omega}$. That is, we define an auxiliary partial computable function $h$, and by the recursion theorem we obtain an index for a total injective computable function $\alpha$ such that $h=J \circ \alpha$. We let $k_{n, m}=\alpha(n, m)$, and instead of saying that we define $h(n, m)$ we say that we define $J\left(k_{n, m}\right)$.

If $n$ enters $\emptyset^{\prime}$ at stage $s$, then let

$$
J\left(k_{n, m}\right)=J_{s}^{C_{s}}(m),
$$

for each $m \leqslant s$ such that $J_{s}^{C_{s}}\left(k_{n, m}\right)$ converges. If there is an $n$ such that the function $m \mapsto g\left(k_{n, m}\right)$ is a DNC function relative to $C$, then we are done. If not, define $f \leqslant_{\mathrm{T}} X \oplus C$ by letting $f(n)$ be the least stage $s$ such that for some $m \leqslant s$, $g\left(k_{n, m}\right)=J_{s}^{C_{s}}(m)$ via a $C$-correct computation. By assumption, $f$ is total.

Suppose that $n \in \emptyset^{\prime}$; say $n$ enters $\emptyset^{\prime}$ at stage $t$. Let $m$ witness that $f(n)=s$. If $t \geqslant s$ then $g\left(k_{n, m}\right)=J^{C}(m)=J\left(k_{n, m}\right)$, contradicting the fact that $g$ is a DNC 
function. Thus, $f$ dominates the settling-time function for $\emptyset^{\prime}$, and so $\emptyset^{\prime} \leqslant_{\mathrm{T}} f \leqslant_{\mathrm{T}}$ $X \oplus C$.

The proof of Theorem 2.5 could be used, with only superficial modification, to give a direct proof of Theorem 2.3. We also note that Theorem 2.5 directly implies Arslanov's completeness criterion.

Corollary 2.6 (Arslanov [1]). If $D$ is a c.e. set of $\mathrm{DNC}$ degree, then $D \equiv_{\mathrm{T}} \emptyset^{\prime}$.

Proof. No set has DNC degree relative to itself, so by Theorem $2.5, D \equiv_{\mathrm{T}} D \oplus D \geqslant_{\mathrm{T}}$ $\emptyset^{\prime}$.

Day and Reimann noticed that Theorem 2.3, together with a relativized version of Arslanov's completeness criterion, shows that every non-low c.e. set joins every $\Delta_{2}^{0}$ set of PA degree to $\emptyset^{\prime}$, and thus proved their Theorem 1.1. The same argument holds if we replace PA by DNC.

First proof of Theorem 1.2. Let $C$ be a c.e. set, let $X \leqslant_{\mathrm{T}} \emptyset^{\prime}$ have DNC degree, and suppose that $\emptyset^{\prime} \Varangle_{\mathrm{T}} X \oplus C$. By Theorem 2.5, $X$ has DNC degree relative to $C$. Since $X \leqslant_{\mathrm{T}} \emptyset^{\prime}, \emptyset^{\prime}$ has DNC degree relative to $C$. Since $\emptyset^{\prime}$ is c.e. relative to $C$, the relativization to $C$ of Arslanov's completeness criterion gives

$$
\emptyset^{\prime} \equiv_{\mathrm{T}} \emptyset^{\prime} \oplus C \geqslant_{\mathrm{T}} C^{\prime},
$$

i.e., $C$ is low.

The proof used Arslanov's completeness criterion to show that if $X \in \Delta_{2}^{0}$ has DNC degree relative to a $\Delta_{2}^{0}$ set $A$ then $A$ is low. We note that the assumption that $A$ be $\Delta_{2}^{0}$ is not necessary. Recall that a set $A$ is generalized low (denoted $\mathrm{GL}_{1}$ ) if $A^{\prime} \equiv_{\mathrm{T}} A \oplus \emptyset^{\prime}$. That is, if when considering the join with $\emptyset^{\prime}$, the Turing jump of $A$ has lowest degree possible. A $\Delta_{2}^{0}$ set is generalized low if and only if it is low.

By relativizing Arslanov's completeness criterion, we get the following:

Proposition 2.7. If some $X \in \Delta_{2}^{0}$ has $\mathrm{DNC}$ degree relative to a set $A \in 2^{\omega}$, then $A$ is generalized low.

Proof. $A \oplus \emptyset^{\prime}$ computes $X$ (since $X$ is $\Delta_{2}^{0}$ ), which has DNC degree relative to $A$. Thus, $A \oplus \emptyset^{\prime}$ has itself DNC degree relative to $A$. But $A \oplus \emptyset^{\prime}$ is also c.e. relative to $A$. By Arslanov's completeness criterion relativized to $A$, we get $A \oplus \emptyset^{\prime} \geqslant_{T} A^{\prime}$.

Nies, Stephan and Terwijn [14] showed that Proposition 2.7 holds if we assume that $X$ is random relative to $A$.

\section{A DiRECT ARGUMENT}

We give a proof of Theorem 1.2 by direct construction. Let $A$ be a non-low c.e. set, and let $f$ be a $\Delta_{2}^{0}$ DNC function; let $\left\langle A_{s}\right\rangle$ be an effective enumeration of $A$, with $A_{s} \neq A_{s+1}$ for all $s$. Let $\left\langle f_{s}\right\rangle$ be a computable approximation for $f$. Also let $\left\langle\emptyset_{s}^{\prime}\right\rangle$ be an effective enumeration of $\emptyset^{\prime}$.

To show that $\emptyset^{\prime}$ is reducible to $A \oplus f$, we need to enumerate, with oracle $A \oplus f$, the complement of $\emptyset^{\prime}$; we build a c.e. operator (also known as an enumeration functional) $\Theta$ for this task. Technically, this is a c.e. set of axioms, which will be triples of the form $(\sigma,(a, b) ; e)$ with the intended interpretation that if $\sigma \prec A$ and $f(a)=b$ then $e \in \Theta(A, f)$. So formally, we let $\Theta(A, f)$ be the collection of numbers $e$ for which there are $\sigma \prec A$ and $a<\omega$ such that $(\sigma,(a, f(a)) ; e) \in \Theta$. Thus, 
$\Theta(A, f)$ is a set c.e. in $A \oplus f$, and we intend it to equal the complement of $\emptyset^{\prime}$. We let $\Theta_{s}$ be the collection of axioms enumerated into $\Theta$ by the end of stage $s$. At stage $s$ we only enumerate into $\Theta$ axioms that pertain to $A_{s}$ and $f_{s}$; if $(\sigma,(a, b) ; e)$ is enumerated into $\Theta$ at stage $s$, then $\sigma \prec A_{s}$ and $f_{s}(a)=b$. We say that we enumerate e into $\Theta(A, f)[s]$ with $A$-use $|\sigma|$ and $f$-use $\{a\}$.

The very basic plan is the following. While a number $e$ is not in $\emptyset^{\prime}$, at various stages $s$ we will want to enumerate $e$ into $\Theta(A, f)$. Of course, sometimes this will be done and then at a later stage $t, e$ will enter $\emptyset^{\prime}$; we then need to undo the enumeration we made at stage $s$. In other words, we need to ensure that either $A$ disagrees with $A_{s}$ or $f$ disagrees with $f_{s}$ on the use of that enumeration. We have one way of forcing such a difference for $f$ : if the $f$-use of the axiom is $\{a\}$, then we can set $J(a)=f_{s}(a)$. Each such input $a$ must correspond to only one stage $s<t$. We do not have to worry about every stage $s<t$, only about those stages $s<t$ for which $A_{s}$ agrees with $A_{t}$ up to the $A$-use, and of course, only about stages $s<t$ at which we enumerated $e$ into $\Theta(A, f)$.

For every stage $s$, let $w_{s}$ be the least number in $A_{s} \backslash A_{s-1}$. At stage $s$ we see that previous versions $A_{r}$ of $A$ were mistaken about $A \uparrow_{w_{s}+1}$. Hence at stage $s$ we are only willing to commit to guessing that $A \uparrow_{w_{s}+1}$ is an initial segment of $A$. The stage $s$ seems to be true at a later stage $t>s$ if that guess was not yet found to be incorrect by stage $t$ : if $A_{s} \uparrow_{w_{s}}=A_{t} \uparrow_{w_{s}}$. We note that if $r<s<t$ and $r$ seems true at stage $t$, then $r$ seems true at stage $s$ as well. A stage $s$ is true if $A_{s} \uparrow_{w_{s}}=A \uparrow_{w_{s}}$; there are infinitely many true stages.

We will only enumerate $e$ into $\Theta(A, f)[s]$ if $e \notin \Theta_{s-1}\left(A_{s}, f_{s}\right)$. This implies that for all $t$, if $e \in \Theta(A, f)[t]$, then there is a unique stage $s \leqslant t$ at which we enumerate $e$ into $\Theta(A, f)[s]$ with an axiom that pertains to $A_{t}, f_{t}$. We say that $s$ is the stage at which we enumerated $e$ into $\Theta(A, f)[t]$. When we enumerate $e$ into $\Theta(A, f)[s]$ then we set the $A$-use of this enumeration to be $w_{s}$. This implies:

Claim 3.1. Suppose that $e \in \Theta_{t-1}\left(A_{t}, f_{t}\right)$, and let $s<t$ be the stage at which this enumeration was made. Then $s$ seems to be true at stage $t$. Indeed, $e \in \Theta_{t-1}\left(A_{t}, f_{t}\right)$ if and only if there is a stage $s<t$ that seems to be true at $t$, at which we enumerate $e$ into $\Theta(A, f)[s]$, such that $f_{t}(a)=f_{s}(a)$, where $\{a\}$ is the use of that enumeration.

For now, denote by $\left\{a_{s}\right\}$ the $f$-use of an enumeration of $e$ into $\Theta(A, f)[s]$. We need to ensure that if $e$ enters $\emptyset^{\prime}$ at some stage $t$, then the numbers $a_{s}$ are distinct, as $s$ ranges over the stages $s<t$ that appear to be true at stage $t$ and at which we enumerated $e$ into $\Theta(A, f)$. This is equivalent to requiring that if $r$ seems true at stage $s$ and at both stages $r$ and $s$ we enumerate $e$, then $a_{r} \neq a_{s}$.

Naïvely, while $e \notin \emptyset^{\prime}$, we would enumerate $e$ into $\Theta(A, f)$ at every stage $s$ at which $e \notin \Theta_{s-1}\left(A_{s}, f_{s}\right)$. But this would force the numbers $a_{s}$ to grow fast. If $e \notin \emptyset^{\prime}$, we need to ensure that $e \in \Theta(A, f)$, and this is equivalent to finding a true stage $s$ at which we enumerate $e$ into $\Theta(A, f)$ such that $f_{s}\left(a_{s}\right)=f\left(a_{s}\right)$. Roughly, we need the $f$-uses $a_{s}$, along the true stages, to not grow faster than the modulus of $f$; if $f_{s}$ does not "catch up" then no enumeration into $\Theta(A, f)$ will be permanent.

So we need to be selective about the choice of stages $s$ at which we enumerate $e$ into $\Theta(A, f)$. This is where we use non-lowness of $A$. We only make such an enumeration at $s$ if we see a new number $i \in A_{s}^{\prime}$.

We use the "hat trick" for the $\Sigma_{2} / \Pi_{2}$ approximation $\left\langle A_{s}^{\prime}\right\rangle_{s<\omega}$ for $A^{\prime}$. At stage $s$, for enumerating $A_{s}^{\prime}$, we only use the part of $A$ that we believe: $A_{s}^{\prime}$ consists of those numbers $i$ for which $J^{A_{s}}(i) \downarrow$ with use at most $w_{s}$. As a result: 
(*) If $s$ appears true at stage $t$ then $A_{s}^{\prime} \subseteq A_{t}^{\prime}$, and $A^{\prime}$ is the union of $A_{s}^{\prime}$ where $s$ ranges over the true stages.

We only enumerate $e$ into $\Theta(A, f)[s]$ if $A_{s}^{\prime} \neq A_{r}^{\prime}$ for every stage $r<s$ that seems true at $s$ and at which we enumerated $e$ into $\Theta(A, f)[r]$. Of course it suffices to check only the last such stage. Roughly, this means that the $f$-uses $a_{s}$ (which in the construction below are actually called $a_{e, i_{s}}$ ) will grow inversely to the modulus of $A^{\prime}$. Since $A^{\prime}>_{\mathrm{T}} \emptyset^{\prime}$ the modulus for $f$ is slower than the modulus for $A^{\prime}$, and so will be able to catch up with the use. This will be formalised by a permitting argument (between $A^{\prime}$ and $\emptyset^{\prime}$ ).

Construction. As in the proof of Theorem 2.5, for each $e<\omega$ we obtain a computable subset $\left\{a_{e, i}: i<\omega\right\}$ of $\operatorname{dom} J$ that we control.

There is no interaction between the parts of the construction that deal with different $e<\omega$. So we fix $e$ and describe the part of the construction that codes $\emptyset^{\prime}(e)$ into $A \oplus f$.

We let $S=S(e)$ be the set of stages $r$ at which we enumerate $e$ into $\Theta(A, f)$. With every stage $s \in S$ we will associate a number $i_{s}=i_{s}(e)$, which we define during the construction; this number will determine the $f$-use of the enumeration we make at stage $s$.

For $s<\omega$, we let $S_{s}=S_{s}(e)$ be the set of stages $r<s$ in $S$ that seem true at stage $s$.

Stage s: If $e \notin \emptyset_{s}^{\prime}$ but $e \notin \Theta_{s-1}\left(A_{s}, f_{s}\right)$, let $r=\max S_{s}\left(r=0\right.$ if $S_{s}$ is empty). If $A_{s}^{\prime} \neq A_{r}^{\prime}$, let $i_{s}=\min A_{s}^{\prime} \backslash A_{r}^{\prime}$. Enumerate $e$ into $\Theta(A, f)[s]$ with $A$-use $w_{s}$ and $f$-use $\left\{a_{e, i_{s}}\right\}$.

If $e \in \emptyset_{s}^{\prime} \backslash \emptyset_{s-1}^{\prime}$ then for all $r \in S_{s}$ we set $J\left(a_{e, i_{r}}\right)=f_{r}\left(a_{e, i_{r}}\right)$. The following claim shows that following this instruction is possible.

Claim 3.2. Let $s<\omega$, and let $r_{0}<r_{1}$ be two stages in $S_{s}$. Then $i_{r_{0}} \neq i_{r_{1}}$.

Proof. The stage $r_{0}$ appears to be true at stage $r_{1}$, so $r_{0} \in S_{r_{1}}$. Let $r=\max S_{r_{1}}$. Note that $r_{0}$ seems true at stage $r$ as well, so $r_{0} \in S_{r} \cup\{r\}$. We have $A_{r_{0}}^{\prime} \subseteq A_{r}^{\prime} \subsetneq$ $A_{r_{1}}^{\prime}$, and $i_{r_{1}} \in A_{r_{1}}^{\prime} \backslash A_{r}^{\prime}$ while $i_{r_{0}} \in A_{r_{0}}^{\prime}$.

Verification. We show that $\Theta(A, f)$ is the complement of $\emptyset^{\prime}$.

Fixing $e$, we observe that Claim 3.1 says that for $s<\omega, e \in \Theta_{s-1}\left(A_{s}, f_{s}\right)$ if and only if there is an $r \in S_{s}$ such that $f_{r}\left(a_{e, i_{r}}\right)=f_{s}\left(a_{e, i_{r}}\right)$.

We let $S_{\omega}$ be the collection of true stages in $S$. Similarly to what we have at finite stages, $e \in \Theta(A, f)$ if and only if there is a stage $r \in S_{\omega}$ such that $f_{r}\left(a_{e, i_{r}}\right)=f\left(a_{e, i_{r}}\right)$.

Lemma 3.3. If $e \in \emptyset^{\prime}$ then $e \notin \Theta(A, f)$.

Proof. Suppose, for a contradiction, that $e \in \Theta(A, f)$; let $r \in S_{\omega}$ be such that $f_{r}\left(a_{e, i_{r}}\right)=f\left(a_{e, i_{r}}\right)$.

Let $s$ be the stage at which $e$ enters $\emptyset^{\prime}$. So $r<s$, and $r$ seems true at stage $s$, so $r \in S_{s}$. Let $i=i_{r}(e)$. At stage $s$ we set $J\left(a_{e, i}\right)=f_{r}\left(a_{e, i}\right)$. Then $f_{r}\left(a_{e, i_{r}}\right)=f\left(a_{e, i_{r}}\right)$ contradicts $f$ being diagonally non-computable.

Lemma 3.4. If $e \notin \emptyset^{\prime}$ then $e \in \Theta(A, f)$.

Proof. The set $S_{\omega}$ is finite if and only if $e \in \Theta(A, f)$. In one direction, this is immediate: if there is an $r \in S_{\omega}$ with $f_{r}\left(a_{e, i_{r}}\right)=f\left(a_{e, i_{r}}\right)$ then once $f_{t}\left(a_{e, i_{r}}\right)$ 
stabilises to $f\left(a_{e, i_{r}}\right)$, for every true stage $s$ we will have $e \in \Theta_{s-1}\left(A_{s}, f_{s}\right)$ and so $s \notin S$, hence not in $S_{\omega}$.

In the other direction, suppose that $S_{\omega}$ is finite; we argue that for some $r \in S_{\omega}$ we have $f_{r}\left(a_{e, i_{r}}\right)=f\left(a_{e, i_{r}}\right)$. If not, from some stage on we have $f_{t}\left(a_{e, i_{r}}\right) \neq f_{r}\left(a_{e, i_{r}}\right)$ for all $r \in S_{\omega}$. For any true stage $s>\max S_{\omega}$ we have $S_{s}=S_{\omega}$. So for sufficiently late true stages $s$ we have $e \notin \Theta_{s-1}\left(A_{s}, f_{s}\right)$. Because $A^{\prime}$ is infinite, for sufficiently late true stages $s$ we have $A_{s}^{\prime} \neq A_{r}^{\prime}$ for $r=\max S_{s}$. So a sufficiently late true stage will be in $S_{\omega}$, for a contradiction.

Suppose that $e \notin \emptyset^{\prime}$; we show that $e \in \Theta(A, f)$. Suppose for a contradiction that $e \notin \Theta(A, f)$, and thus that $S_{\omega}$ is infinite. Fix a $j \in A^{\prime}$, and let $t \in S_{\omega}$ be least with $j \in A_{t}^{\prime}$. Then $i_{t} \leqslant j$, and since $e \notin \Theta(A, f)$, we see that $f_{t}\left(a_{e, i_{t}}\right) \neq f\left(a_{e, i_{t}}\right)$.

Since $\emptyset^{\prime}$ can compute $S_{\omega}$ and can tell if there is a future change in $f_{s}\left(a_{e, k}\right)$, for any $j, \emptyset^{\prime}$ can effectively locate a stage $s \in S_{\omega}$ such that $f_{t}\left(a_{e, k}\right)=f\left(a_{e, k}\right)$ for all $t \geqslant s$ and all $k \leqslant j$. By the argument of the previous paragraph, $j \in A^{\prime}$ if and only if $j \in A_{s}^{\prime}$. Thus $\emptyset^{\prime}$ can compute $A^{\prime}$, a contradiction.

\section{Kolmogorov COMPLEXITY}

It is easy to see that there is a function $g \equiv_{\mathrm{T}} \emptyset^{\prime}$ such that every function that dominates $g$ computes $\emptyset^{\prime}$; for example, we can take $g$ to be the settling-time function for $\emptyset^{\prime}$. We can also take a so-called "busy-beaver" function.

Lemma 4.1. Let $g(n)=\min \{t \in \mathbb{N}: \forall s \geqslant t(K(s)>n)\}$. Any function dominating $g$ computes $\emptyset^{\prime}$.

Proof. If $n$ enters $\emptyset^{\prime}$ at stage $s$ then $K(s) \leqslant^{+} K(n)$. Let $c$ be the constant witnessing this inequality. Then $n \in \emptyset^{\prime}$ if and only if $n \in \emptyset_{g(n+c)}^{\prime}$.

Kjos-Hanssen, Merkle and Stephan [8] showed an elegant characterization of DNC degrees in terms of Kolmogorov complexity.

Proposition 4.2 (Kjos-Hanssen, Merkle, Stephan). A set A has DNC degree if and only if it computes a sequence $\left\langle x_{n}\right\rangle_{n<\omega}$ such that $K\left(x_{n}\right) \geqslant{ }^{+} n$.

Proof. Kjos-Hanssen, Merkle and Stephan actually show this result with plain Kolmogorov complexity $C$ instead of prefix-free Kolmogorov complexity. The argument given in [8] applies to prefix-free complexity as well. For completeness of presentation we recall that argument.

First suppose that $A$ computes a sequence $\left\langle x_{n}\right\rangle_{n<\omega}$ with $K\left(x_{n}\right) \geqslant n-c$ for some $c$. As we discussed in the proof of Proposition 2.4, if $J(n)$ is defined, then $K(J(n))<2 \log n+d$ for some fixed constant $d$. Let $e$ be a constant such that $n-c+e>2 \log n+d$ for all $n$. The function $f$ defined by $f(n)=x_{n+e}$ satisfies, by assumption on the sequence $\left\langle x_{n}\right\rangle_{n<\omega}, K(f(n))>n-c+e>2 \log n+d$ and thus $f(n) \neq J(n)$ for all $n$.

Conversely, let $A$ be of DNC degree and $f \leqslant_{T} A$ a DNC function. By the recursion theorem, we control a computable subset $\left\{a_{\sigma}: \sigma \in 2^{<\omega}\right\}$ of the domain of the universal function $J$. Let $\mathbb{U}$ be the universal prefix-free machine, and now we think of the elements in the range of $\mathbb{U}$ as strings in $\omega^{<\omega}$. For $\sigma \in \operatorname{dom} \mathbb{U}$, if $|\mathbb{U}(\sigma)|>a_{\sigma}$ then let $J\left(a_{\sigma}\right)=(\mathbb{U}(\sigma))\left(a_{\sigma}\right)$; otherwise, $J\left(a_{\sigma}\right) \uparrow$. 
For $n<\omega$, take $\tau_{n} \prec f$ such that $\left|\tau_{n}\right|>a_{\sigma}$ for all $|\sigma|<n$. Then $K\left(\tau_{n}\right) \geqslant n$, because we diagonalized against descriptions of length below $n$ : if $|\sigma|<n$ is in $\operatorname{dom} \mathbb{U}$, then either $|\mathbb{U}(\sigma)|<\left|\tau_{n}\right|$, or

$$
\tau_{n}\left(a_{\sigma}\right)=f\left(a_{\sigma}\right) \neq J\left(a_{\sigma}\right)=\mathbb{U}(\sigma)\left(a_{\sigma}\right) ;
$$

in either case, $\tau_{n} \neq \mathbb{U}(\sigma)$.

Using Proposition 4.2, we can translate the arguments of Section 2 to the language of Kolmogorov complexity. The proofs we obtain have a different flavor. The following proposition is essentially equivalent to Theorem 2.5.

Proposition 4.3. Let $A$ be a c.e. set. Suppose that for every $\Delta_{2}^{0}$ sequence $\left\langle x_{n}\right\rangle_{n<\omega}$ we have $K^{A}\left(x_{n}\right) \not{ }^{+} n$. Then $A$ joins every $\Delta_{2}^{0}$ DNC function to $\emptyset^{\prime}$.

Proof. Let $f$ be a $\Delta_{2}^{0}$ DNC function. By Proposition 4.2, $f$ computes a sequence $\left\langle x_{n}\right\rangle_{n<\omega}$ such that $K\left(x_{n}\right) \geqslant n$ for all $n$. We fix such a sequence.

We show how to use the oracle $A \oplus\left\langle x_{n}\right\rangle$ to dominate the function $g$ of Lemma 4.1. As the overgraph of $K^{A}$ is $A$-enumerable, given $e<\omega$, we can $A \oplus\left\langle x_{n}\right\rangle$-effectively find an $n=n(e)$ such that $K^{A}\left(x_{n}\right)<n-e$. Having found $n$, we let $t(e)$ be the least stage $t$ at which we see that $K^{A}\left(x_{n}\right)[t]<n-e$ via an $A$-correct computation. We claim that for some constant $d$, which does not depend on $e$, we have $K(s) \geqslant e-d$ for all $s \geqslant t(e)$. Since $e \mapsto t(e+d)$ is $(A \oplus X)$-computable and by the above dominates the function $g$ of Lemma 4.1, this will be sufficient to get $A \oplus X \geqslant_{T} \emptyset^{\prime}$.

Let $s \geqslant t(e)$, and let $n=n(e)$. Then $K^{A}\left(x_{n}\right)[s]<n-e$. We claim that $K\left(x_{n}\right) \leqslant^{+}(n-e)+K(s)$, with the constant not depending on $s$ or $e$. Suppose that $\mathbb{U}^{A}(\sigma)[s] \downarrow=x_{n}$ with $|\sigma|<n-e$. Then effectively, given a description for $s$ concatenated with $\sigma$, we first find $s$ and $\sigma$, and output $\mathbb{U}^{A}(\sigma)[s]$.

Finally, since $K\left(x_{n}\right) \geqslant n$, we see immediately that $K\left(x_{n}\right) \leqslant(n-e)+K(s)$ implies that $K(s) \geqslant+e$ as required.

Combining Proposition 4.3 with the following proposition gives us, essentially, the first proof of Theorem 1.2. The proposition is very similar to Proposition 2.7. However, here we give a direct argument, inspired by the one in Section 3 .

Proposition 4.4. Let $A \in 2^{\omega}$ be a set that is not generalized low. Then for any $\Delta_{2}^{0}$ sequence $\left\langle x_{n}\right\rangle_{n<\omega}, K^{A}\left(x_{n}\right) \not{ }^{+} n$.

Proof. Fix a $\Delta_{2}^{0}$ sequence $\left\langle x_{n}\right\rangle_{n<\omega}$. We need to show that for all $e$ there is an $n$ such $K^{A}\left(x_{n}\right) \leqslant n-e$. Via the coding theorem, it is equivalent to build a lower $A$-c.e. discrete measure $m$ such that for all $e$ there is an $n$ such that $m\left(x_{n}\right) \geqslant 2^{e-n}$. This is done independently for each $e$. That is, uniformly in $e$, we define a lower $A$-c.e. discrete measure $m_{e}$, whose total weight $\sum_{k<\omega} m_{e}(k)$ is bounded by $2^{-e}$; we then let $m=\sum_{e<\omega} m_{e}$. Fulfilling the requirement for $e$ will be done by ensuring that $m_{e}\left(x_{n}\right) \geqslant 2^{e-n}$ for some $n$. We use permitting between $A^{\prime}$ and $A \oplus \emptyset^{\prime}$ to show that attempts at compression must sometimes succeed.

Let $\left\langle x_{n, s}\right\rangle$ be a computable approximation for $\left\langle x_{n}\right\rangle$. Also, fix an $A$-computable enumeration $\left\langle A_{s}^{\prime}\right\rangle_{s<\omega}$ of $A^{\prime}$.

We define $m_{e}$ as follows. If $n>2 e$ enters $A^{\prime}$ at stage $s$, then we increase $m_{e}\left(x_{n, s}\right)$ by $2^{e-n}$.

First we observe that indeed the total mass of $m_{e}$ is bounded by $2^{-e}$. Each number $n$ enters $A^{\prime}$ at most once, and so the total mass is bounded by $2^{e} \cdot \sum_{n>2 e} 2^{-n} \leqslant$ $2^{e-2 e}$ as required. 
Next we show that there is an $n$ such that $m_{e}\left(x_{n}\right) \geqslant 2^{e-n}$. Suppose not; we show how to compute $A^{\prime}$ from $A \oplus \emptyset^{\prime}$. To decide if $n \in A^{\prime}$, find a stage $s$ such that $x_{n, t}=x_{n}$ for all $t \geqslant s$. We claim that $n \in A^{\prime}$ if and only if $n \in A_{s}^{\prime}$. For if $n$ enters $A^{\prime}$ at a stage $t>s$, then at that stage we add a mass of $2^{e-n}$ to $m_{e}\left(x_{n}\right)$, which contradicts our assumption.

Weaker variants of Theorem 1.2 can be proved quickly using Proposition 4.3. We give an example. Recall that a set $A$ is LR-hard if and only if $K^{A} \leqslant K^{+}$. There are incomplete, LR-hard c.e. sets.

Theorem 4.5. Every LR-hard c.e. set joins every $\Delta_{2}^{0}$ DNC function to $\emptyset^{\prime}$.

Proof. Let $A$ be LR-hard. We verify the condition of Proposition 4.3. Let $\left\langle x_{n}\right\rangle$ be $\Delta_{2}^{0}$. Since $\emptyset^{\prime}$ computes the sequence $\left\langle x_{n}\right\rangle$, we have $K^{\emptyset^{\prime}}\left(x_{n}\right) \leqslant{ }^{+} K^{\emptyset^{\prime}}(n) \leqslant 2 \log _{2} n$. Hence $K^{A}\left(x_{n}\right) \leqslant+2 \log _{2} n$; and $\lim _{n \rightarrow \infty}(n-2 \log n)=\infty$.

A similar argument can be extended to all superhigh sets, and a bit more. The condition we use is $K^{A} \leqslant^{+} h\left(K^{\emptyset^{\prime}}\right)$ for a computable or even $\Delta_{2}^{0}$ function $h$.

\section{REFERENCES}

[1] Marat M. Arslanov. Some generalizations of a fixed-point theorem. Izv. Vyssh. Uchebn. Zaved. Mat., 5:9-16, 1981. English translation: Soviet Math. (Iz. VUZ), 1981, 25:5, 1-10. 2, 6

[2] Adam R. Day and Joseph S. Miller. Randomness for non-computable measures. Transactions of the American Mathematical Society, to appear. 4

[3] Adam R. Day and Jan Reimann. Independence, relative randomness, and PA degrees. To apeear in Notre Dame Journal of Formal Logic, 201x. 2, 4

[4] R. Downey and D. Hirschfeldt. Algorithmic randomness and complexity. Springer-Verlag, Berlin, 2010. 855 pages. 5

[5] Denis R. Hirschfeldt, André Nies, and Frank Stephan. Using random sets as oracles. Journal of the London Mathematical Society (2), 75(3):610-622, 2007. 5

[6] Carl G. Jockusch, Jr. Degrees of functions with no fixed points. In Logic, methodology and philosophy of science, VIII (Moscow, 1987), volume 126 of Stud. Logic Found. Math., pages 191-201. North-Holland, Amsterdam, 1989. 2

[7] Carl Jockusch Jr., Manuel Lerman, Robert I. Soare, and Robert Solovay. Recursively enumerable sets modulo iterated jumps and extensions of Arslanov's completeness criterion. Journal of Symbolic Logic, 54(4):1288-1323, 1989. 2

[8] Bjørn Kjos-Hanssen, Wolfgang Merkle, and Frank Stephan. Kolmogorov complexity and the recursion theorem. Trans. Amer. Math. Soc., 363(10):5465-5480, 2011. 9

[9] Antonín Kučera. Measure, $\Pi_{1}^{0}$-classes and complete extensions of PA. In Recursion theory week (Oberwolfach, 1984), volume 1141 of Lecture Notes in Math., pages 245-259. Springer, Berlin, 1985. 5

[10] Antonin Kučera. An alternative, priority-free, solution to Post's problem. In Mathematical Foundations of Computer Science, 1986 (Bratislava, 1986), volume 233 of Lecture Notes in Comput. Sci., pages 493-500. Springer, Berlin, 1986. 2

[11] Antonin Kučera. On the role of $\mathbf{0}^{\prime}$ in recursion theory. In Logic colloquium '86 (Hull, 1986), volume 124 of Stud. Logic Found. Math., pages 133-141. North-Holland, Amsterdam, 1988. 2

[12] A. Nies. Computability and randomness, volume 51 of Oxford Logic Guides. Oxford University Press, Oxford, 2009. 5

[13] André Nies. Lowness properties and randomness. Advances in Mathematics, 197(1):274-305, 2005. 5

[14] André Nies, Frank Stephan, and Sebastiaan A. Terwijn. Randomness, relativization and Turing degrees. J. Symbolic Logic, 70(2):515-535, 2005. 6

[15] Stephen G. Simpson. Degrees of unsolvability: A survey of results. In J. Barwise, editor, Handbook of Mathematical Logic, pages 631-652. North-Holland, Amsterdam, 1977. 5 
[16] Robert I. Soare. Computational complexity, speedable and levelable sets. Journal of Symbolic Logic, 42(4):545-563, 1977. 1

[17] Robert I. Soare. Recursively enumerable sets and degrees. Perspectives in Mathematical Logic. Springer-Verlag, Berlin, 1987. A study of computable functions and computably generated sets. 2

LiAFA, CNRS \& University of Paris 7, Paris, France

E-mail address: laurent.bienvenu@liafa.univ-paris-diderot.fr

School of Mathematics, Statistics and Operations Research, Victoria University of Wellington, Wellington, New Zealand

E-mail address: greenberg@msor.vuw.az.nz

Charles University in Prague, Faculty of Mathematics and Physics, Prague, Czech REPUBLIC

E-mail address: kucera@mbox.ms.mff.cuni.cz

Department of Mathematics, University of Wisconsin, Madison, Wi 53706-1388, USA

E-mail address: jmiller@math.wisc.edu

Department of Computer Science, University of Auckland, Private Bag 92019, AuckLAND, NEW ZEALAND

E-mail address: andre@cs.auckland.ac.nz

Kurt Gödel Research Center for Mathematical Logic, University of Vienna, Vienna, Austria

E-mail address: turetsd4@univie.ac.at 